\title{
Treatment with glutamine is associated with down-regulation of Toll-like receptor- 4 and myeloid differentiation factor 88 expression and decrease in intestinal mucosal injury caused by lipopolysaccharide endotoxaemia in a rat
}

\author{
A. Kessel, ${ }^{\star}$ E. Toubi, ${ }^{\star}$ E. Pavlotzky, ${ }^{\star}$ \\ J. Mogilner, ${ }^{\dagger}$ A. G. Coran, ${ }^{\ddagger}$ M. Lurie, \\ R. Karry ${ }^{\dagger}$ and I. Sukhotnik ${ }^{\dagger}$ \\ ${ }^{*}$ Allergy and Clinical Immunology, Department \\ of ${ }^{\dagger}$ Pediatric Surgery and ${ }^{\S}$ Pathology, Bnai Zion \\ Medical Center, The Bruce Rappaport Faculty of \\ Medicine, Technion-Israel Institute of Technology, \\ Haifa, Israel, and "Section of Pediatric Surgery, \\ C. S. Mott Children's Hospital and University of \\ Michigan Medical School, Ann Arbor, MI, USA
}

Accepted for publication 9 November 2007 Correspondence: A. Kessel, Division of Allergy and Clinical Immunology, Bnai-Zion Medical Center, 47 Golomb Street, PO Box 4940, Haifa 31048, Israel.

E-mail: aharon.kessel@b-zion.org.il

\begin{abstract}
Summary
Recent evidence suggests that lipopolysaccharide (LPS) endotoxaemia in a rat causes significant mucosal injury. Our objective was to determine the effects of glutamine (Gln) on Toll-like receptor 4 (TLR-4), myeloid differentiation factor 88 (Myd88) and tumour necrosis factor (TNF)- $\alpha$ receptor-associated factor 6 (TRAF6) expression in intestinal mucosa following LPS endotoxaemia in a rat. For this purpose, male Sprague-Dawley rats were assigned randomly to one of three experimental groups of 10 rats each: (i) control rats underwent intraperitoneal (i.p.) injection of sterile saline once a day; (ii) rats were treated with LPS given i.p. once a day at a dose of $10 \mathrm{mg} / \mathrm{kg}$ for $48 \mathrm{~h}$ (two doses); and (iii) rats were pretreated with oral Gln given in drinking water (2\%) $48 \mathrm{~h}$ before and following injection of LPS. Intestinal mucosal parameters, enterocyte proliferation and apoptosis were determined at death. TLR-4 and MyD88 mRNA expression was measured with reverse transcriptionpolymerase chain reaction (RT-PCR). TLR- 4 and MyD88 protein expression were analysed by Western immunoblotting. We observed a statistically significant $(P<0.05)$ decrease in mucosal weight, mucosal DNA and enterocyte proliferation and a significant increase in enterocyte apoptosis in rat intestine, following LPS administration. These changes were attenuated significantly by dietary Gln. Expression of TLR-4, MyD88 and TRAF6 mRNA in the mucosal ileum was significantly higher in LPS rats versus control rats $(P=0.0006$, $P=0.0015, P=0.03$, respectively) as well as TLR-4 and MyD88 protein expression. The administration of Gln reduced significantly the expression of TLR-4, MyD88 and TRAF6 $(P=0.023, P=0.014, P=0.035$, respectively) mRNA as well as TLR-4 and MyD88 protein expression in ileum compared to LPS animals. We did not find a significant change in the expression of TLR-4, MyD88 or TRAF6 in the jejunum of different groups. We conclude that treatment with Gln was associated with down-regulation of TLR-4, MyD88 and TRAF6 expression and concomitant decrease in intestinal mucosal injury caused by LPS endotoxaemia in a rat.
\end{abstract}

Keywords: endotoxaemia, glutamine, lipopolysaccharide, mucosal injury, MyD88, Toll-like receptor 4

\section{Introduction}

Toll-like receptors (TLRs) are an ancient conserved receptor family that regulate anti-microbial host defence in plants, invertebrates and mammals [1]. Individual TLRs recognize distinct pathogen-associated molecular patterns (PAMPs) that have been conserved evolutionarily in specific classes of microbes. Interaction of PAMPs with TLRs triggers a complex signalling pathway that leads to the activation of the immune system. There are more than 10 identified members of the TLR family, with well-defined specificities to various components of bacteria, viruses or fungi [2]. The best-characterized member of this family is Toll-like receptor 4 (TLR-4), the receptor for lipopolysaccharide (LPS), which is the best-known and first-discovered bacterial cell wall component that can elicit cellular responses. TLR-4 is responsible for the recognition of bacterial endotoxin or LPS and for initiation of Gram-negative bacillary septic 
shock syndrome $[3,4]$. Interaction between LPS and TLR-4 leads to the formation of an LPS signalling complex consisting of surface molecules, such as CD14 and MD2, as well as intracellular adaptor molecules, including myeloid differentiation primary response gene 88 (MyD88) and tumour necrosis factor (TNF)- $\alpha$ receptor association factor 6 (TRAF6), and activation of transcription factors such as nuclear factor $\kappa \mathrm{B}(\mathrm{NF \kappa B})$, which then induce activation of the inflammatory genes, such as TNF- $\alpha$, interleukin (IL)-1, IL-6 and IL-8 [5].

Extensive studies in various experimental models have established that intestinal mucosal injury impairs gut barrier function and leads to bacterial translocation of bacteria and endotoxin across the intestinal mucosa, which could result in the systemic inflammatory response syndrome and multiple organ failure [6]. However, in a recent study we have demonstrated that LPS endotoxaemia itself impairs the integrity of the gastrointestinal mucosa [7]. This mechanism may be responsible for bacterial translocation and development of the systemic inflammatory response in septic patients. Therefore, understanding of the mechanisms by which LPS impairs intestinal integrity (e.g. the TLR-4 pathway) as well as identification of those factors that prevent intestinal mucosal injury might suggest new therapeutic strategies in maintaining gut barrier function and in improving the outcome in patients suffering from endotoxaemia and sepsis.

Glutamine (Gln) is a non-essential amino acid which is also considered an essential nutrient during major injury or severe illness. It has been demonstrated that a rapid depletion of Gln stores occurs during critical illness [8-10]. Gln has been shown to be beneficial in the prevention of infectious morbidity and mortality in seriously ill patients, due in part to its ability to maintain the integrity of intestinal mucosal epithelium [11-14].

Several possible mechanisms have been described to explain the positive effects of Gln on intestine. These include replication of enterocytes, maintenance of gutassociated lymphoid tissue function and cellularity. It has also been suggested that Gln influences replication of immune cells, $\mathrm{T}$ helper function and responsiveness and synthesis of immunoglobulin A (IgA) [15]. Recently, Uehara et al. demonstrated that Gln pretreatment improves intestinal tissue injury significantly in a rat model of LPS endotoxaemia. Gln exerts a protective effect by its site-specific induction of haem oxygenase- 1 in the mucosal cells in the ileum and the colon and attenuates tissue inflammation, apoptotic cell death and decreases mortality in rats [16]. We have shown recently that oral Gln prevents gut mucosal injury and improves mucosal recovery following lipopolysaccharide endotoxaemia in a rat [17].

In this study, we examined the effect of Gln administration on TLR-4, MyD88 and TRAF6 mRNA expression in small intestine following LPS endotoxaemia in a rat.

\section{Materials and methods}

\section{Animals}

The experiment and animal care were conducted in compliance with the guidelines established by the Guide for the Care and Use of Laboratory Animals, Rappaport Faculty of Medicine, Technion (Haifa, Israel).

Male Sprague-Dawley rats weighing 250-280 g were utilized for this study. Animals were housed in individual wirebottomed Plexiglas cages kept at $21^{\circ} \mathrm{C}$ on 12 -h day-night cycles for at least 4 days with free access to water and were pair-fed with a standard chow.

\section{Experimental design}

Animals were assigned randomly to one of three experimental groups of 10 rats each: (1) control rats (group A) underwent intraperitoneal (i.p.) injection of sterile saline once a day; (2) LPS rats (group B) rats were treated with LPS given i.p. once a day at a dose of $10 \mathrm{mg} / \mathrm{kg}$ for $48 \mathrm{~h}$ (two doses); and (3) LPS-Gln (group C) rats were pretreated with oral Gln given in drinking water (2\%) $48 \mathrm{~h}$ before and following injection of LPS. Twenty-four $h$ following the last LPS injection, animals were anaesthetized with i.p. sodium pentobarbital (45 mg/kg) and were killed by open pneumothorax.

\section{Intestinal mucosal parameters}

The small bowel was excised quickly, washed with cold isotonic saline and divided into two segments: proximal jejunum and terminal ileum. Each segment was weighed and cut longitudinally. Mucosa was scraped using a microspatula, collected and weighed. DNA was extracted from the mucosa of jejunum and ileum using TRIzol reagent, as described by Chomczynski [18]. Quantitization of DNA was performed by spectrophotometry using A260 value (one A260 unit equals $50 \mu \mathrm{g}$ of double-stranded DNA) and calculated as $\mu \mathrm{g} / \mathrm{cm}$ bowel length/100 g body weight.

\section{Crypt cell proliferation and villus cell apoptosis}

Standard 5-bromodeoxyuridine (5-BrdU) labelling reagent (Zymed Laboratories, Inc., San Francisco, CA, USA) was injected i.p. at a dose of $1 \mathrm{ml} / 100 \mathrm{~g}$ body weight $2 \mathrm{~h}$ before sacrifice. Crypt cell proliferation was assessed using biotinylated monoclonal anti-BrdU antibody system provided in kit form (Zymed Laboratories, Inc.). An index of proliferation was determined as the ratio of crypt cells staining positively for BrdU per 10 crypts.

Immunohistochemistry for caspase-3 (caspase-3 cleaved concentrated polyclonal antibody; dilution 1:100; Biocare Medical, Walnut Greek, CA, USA) was performed for identification of apoptotic cells using a combination of the streptovidin-biotin-peroxidase method and microwave 
Table 1. Data for first and second antibodies used for Western blot analysis.

\begin{tabular}{lccc}
\hline Second antibody (dilution) & Size $(\mathrm{kDa})$ & Manufacturer and catalogue number & Antibody (dilution) \\
\hline Mouse monoclonal (1:7500) & 43 & Sigma Aldrich & $\beta$-actin (1:2000) \\
Goat polyclonal (1:7500) & 33 & Santa Cruz sc-8196 & MyD88 (N-19) (1:300) \\
Mouse IgG2b (1:3000) & 88 & Imgenex (Almog) IMG-5031A & TLR-4 (1:200) \\
\hline
\end{tabular}

MyD88: myeloid differentiation factor 88; TLR-4: Toll-like receptor 4.

antigen retrieval on formalin-fixed, paraffin-embedded tissues according to the manufacturer's protocols. The apoptotic index (AI) was defined as the number of apoptotic caspase-3-positive cells per 10 villi.

All measurements were performed by a qualified pathologist blinded as to the source of intestinal tissue.

\section{TLR-4, MyD88 and TRAF6 mRNA expression by semiquantitative reverse transcription-polymerase chain reaction (RT-PCR) analysis}

Total RNA from ileal and jejunal rat epithelial cells was isolated with MasterPure ${ }^{\text {mi }}$ (Epicentre, Madison, WI, USA). cDNA was generated from $1 \mu \mathrm{g}$ of total RNA using reverse transcriptase (Reverse-iT $\mathrm{T}^{\mathrm{TN}} \mathrm{ABgene}$, Surrey, UK) and random decamers (ABgene). RT products were subjected to polymerase chain reaction (PCR) amplification with GoTaq Green Master Mix (Promega, Madison, WI, USA). The annealing temperature was $54^{\circ} \mathrm{C}$. All primers were purchased from Genosys, Sigma, Rehovot, Israel. The cDNA products were separated on $2 \%$ agarose gel containing ethidium bromide. The bands were visualized and photographed using ultraviolet (UV) transilluminator and analysed by Tina software. $\beta$-actin $C D N A$ product was used as a standard to equivalent levels of total RNA subjected to RT-PCR and used to normalize the band intensity of TLR-4 MyD88 and TRAF6.

\section{Western blot analysis}

For separation of total protein, frozen specimens were homogenized in $50 \mathrm{mM}$ Tris buffer $\mathrm{pH} 7.4$ containing $150 \mathrm{mM} \mathrm{NaCl}, 5 \mathrm{mM}$ ethylenediamine tetraacetic acid (EDTA), $0.5 \%$ NP40 and TM protease inhibitor cocktail. Following centrifugation (16 $000 \mathrm{~g}, 10 \mathrm{~min})$, the supernatant (100 $\mu \mathrm{g}$ total protein, according to calibration) was diluted $1: 1$ in electrophoresis sample buffer containing $20 \%(\mathrm{v} / \mathrm{v})$ glycerol, $4 \%(\mathrm{w} / \mathrm{v})$ sodium dodecyl sulphate (SDS), $250 \mathrm{mM}$ Tris-HCl, $\mathrm{pH} 6 \cdot 8, \quad 10 \% \quad(\mathrm{v} / \mathrm{v})$ 2-mercaptoethanol and $0.5 \mathrm{mg} / \mathrm{ml}$ bromophenol blue. The protein sample was separated on 10\% SDS acrylamide gel and transferred to a nitrocellulose membrane. Quality of transfer was assayed by Ponceau staining. Following blocking [Tween-Tris-buffered saline (TBS) containing $2 \%$ bovine serum albumin (BSA)] of non-specific binding sites, membranes were incubated at $4^{\circ} \mathrm{C}$ overnight with primary antibody. After washing, blots were incubated for $1 \mathrm{~h}$ at room temperature with secondary antibody in Tween-TBS (in 0.5\% Tween). Data regarding specific antibody used, molecular weight, dilution, and secondary antibody are summarized in Table 1 . The blots were then developed with Amersham's enhanced chemiluminescence (ECL) reagent and exposed to XLS Kodak film for 20-30 s. Protein levels were analysed three times for each sample. A single batch of protein of rat intestine in three different concentrations was used as a positive control and for normalization. Protein concentration was measured using Bradford reagent (Bio-Rad Laboratories Ltd., Rishon Le Zion, Israel). The results are expressed as the ratio of target protein over $\beta$-actin protein expression.

\section{Statistical analysis}

The data was analysed by one-way analysis of variance (ANOva) following unpaired Student's $t$-test with Bonferroni's multiple comparison tests. Differences at $P=0.05$ were considered statistically significant.

\section{Results}

\section{Intestinal mucosal parameters}

LPS rats (group B) had a significant decrease in bowel weight in jejunum $(15 \%, P<0 \cdot 05)$, mucosal weight in jejunum $(33 \%, P<0.05)$ and ileum $(43 \%, P<0.05)$, mucosal DNA in jejunum $(54 \%, P<0.05)$ and ileum $(53 \%, P<0.05)$ compared to control animals (group A) (Table 2). Following oral Gln administration, LPS-Gln rats (group C) demonstrated a significant increase in jejunal $(18 \%, P<0.05)$ and ileal $(18 \%$, $P<0.05)$ bowel weight, ileal mucosal weight $(44 \%, P<0.05)$ and a twofold increase in jejunal $(P<0.05)$ and ileal $(P<0.05)$ mucosal DNA content compared to LPSuntreated animals (group B).

\section{Enterocyte proliferation and apoptosis}

Enterocyte proliferation decreased significantly in LPS rats (group B) in both jejunum $(18 \%, P<0.05)$ and ileum $(22 \%$, $P<0.05)$ compared to control animals (group B) (Table 2). Pretreatment with oral Gln (group C) led to a significant increase in the enterocyte proliferation rate in jejunum $(21 \%, P<0.05)$ and ileum $(14 \%, P<0.05)$ compared to LPS animals (group B). The number of apoptotic cells increased significantly in LPS rats (group B) in jejunum (2.5-fold, $P<0.05)$ and ileum $(2.5$-fold, $P<0.05)$ compared to control animals (Table 1). LPS-Gln rats (group C) showed a trend 
Table 2. Effect of lipopolysaccharide (LPS) endotoxaemia and treatment with glutamine on intestinal mucosal parameters.

\begin{tabular}{|c|c|c|c|}
\hline Parameters & Control & LPS & LPS-Gln \\
\hline \multicolumn{4}{|c|}{ Bowel weight (mg/cm/100 g) } \\
\hline Jejunum & $20 \pm 0 \cdot 3$ & $17 \pm 1^{\star}$ & $20 \pm 0 \cdot 6^{\dagger}$ \\
\hline Ileum & $18 \pm 1$ & $17 \pm 1$ & $20 \pm 0 \cdot 7^{\star 1}$ \\
\hline \multicolumn{4}{|c|}{ Mucosal weight $(\mathrm{mg} / \mathrm{cm} / 100 \mathrm{~g})$} \\
\hline Jejunum & $9 \pm 0 \cdot 5$ & $6 \pm 0 \cdot 5^{*}$ & $7 \pm 0 \cdot 4^{*}$ \\
\hline Ileum & $8 \pm 0 \cdot 6$ & $4 \cdot 6 \pm 0 \cdot 3^{*}$ & $6 \cdot 6 \pm 0 \cdot 4^{* \dagger}$ \\
\hline \multicolumn{4}{|c|}{ Mucosal DNA ( $\mu \mathrm{g} / \mathrm{cm} / 100 \mathrm{~g})$} \\
\hline Jejunum & $12 \cdot 3 \pm 0 \cdot 2$ & $5 \cdot 7 \pm 1 \cdot 2^{*}$ & $11 \cdot 7 \pm 0 \cdot 4^{\dagger}$ \\
\hline Ileum & $11 \cdot 7 \pm 1 \cdot 1$ & $5 \cdot 5 \pm 1 \cdot 4^{*}$ & $10 \cdot 5 \pm 0 \cdot 4^{\dagger}$ \\
\hline \multicolumn{4}{|c|}{ Cell proliferation (BrdU-positive cells/10 crypts) } \\
\hline Jejunum & $142 \pm 10$ & $116 \pm 2^{*}$ & $140 \pm 3^{\dagger}$ \\
\hline Ileum & $156 \pm 14$ & $121 \pm 6^{*}$ & $138 \pm 4^{\dagger}$ \\
\hline \multicolumn{4}{|c|}{ Cell proliferation (apoptotic cells/10 villi) } \\
\hline Jejunum & $1 \cdot 3 \pm 0 \cdot 2$ & $3 \cdot 2 \pm 0 \cdot 7^{*}$ & $2 \cdot 7 \pm 0 \cdot 5^{*}$ \\
\hline Ileum & $1 \cdot 3 \pm 0 \cdot 5$ & $3 \cdot 0 \pm 0 \cdot 6^{*}$ & $2 \cdot 3 \pm 0 \cdot 1$ \\
\hline
\end{tabular}

${ }^{\star} P<0.05$ LPS versus control $;{ }^{\dagger} P<0.05$ LPS-Gln versus LPS. LPS: rats treated with LPS; LPS-Gln: rats treated with LPS and glutamine; BrdU: bromodeoxyuridine. towards a decrease in the enterocyte apoptosis in jejunum and ileum compared to LPS rats (group B); however, this trend did not achieve statistical significance (Fig. 1).

\section{TLR-4, MyD88 and TRAF6 mRNA expression}

LPS rats (group B) showed a significant increase in TLR-4 mRNA expression versus controls $(P=0.0006)$ in ileum and trend towards an increase in TLR-4 mRNA expression in jejunum; however, this trend was not statistically significant (Fig. 2). Treatment with Gln (group C) resulted in a significant decrease in ileal TLR-4 mRNA expression (versus LPS rats, group B) $(P=0.023)$ and a trend towards a decrease in jejunum TLR-4 mRNA expression; however, this trend did not achieve statistical significance. Changes in MyD88 mRNA expression were similar to those of TLR-4 mRNA expression (Fig. 3). LPS endotoxaemia (group B) resulted in a significant increase in MyD88 mRNA expression in ileum compared to control rats (group A) $(P=0 \cdot 0015)$. LPS-Gln rats (group C) showed a significant decrease (versus LPS rats $)$ in MyD88 mRNA expression in ileum $(P=0.014)$ and a
Fig. 1. Effect of lipopolysaccharide (LPS) endotoxaemia and oral glutamine on crypt cell proliferation and enterocyte apoptosis; 5-bromodeoxyuridine (5-BrdU) incorporation into proliferating jejunal and ileal crypt cells was detected with a goat anti-BrdU antibody. The representative sections (on the left side) demonstrate that cell proliferation decreased following LPS compared to sham animals. Following administration of oral glutamine, LPS rats demonstrated a significant increase in a number of proliferating cells compared to LPS non-treated animals.

Immunohistochemistry for caspase-3 was used to determine enterocyte apoptosis. The representative sections (on the right side) demonstrate that the number of apoptotic cells increased significantly following LPS compared to control animals and decreased significantly after treatment with glutamine. Gln: glutamine.

\section{Proliferation}
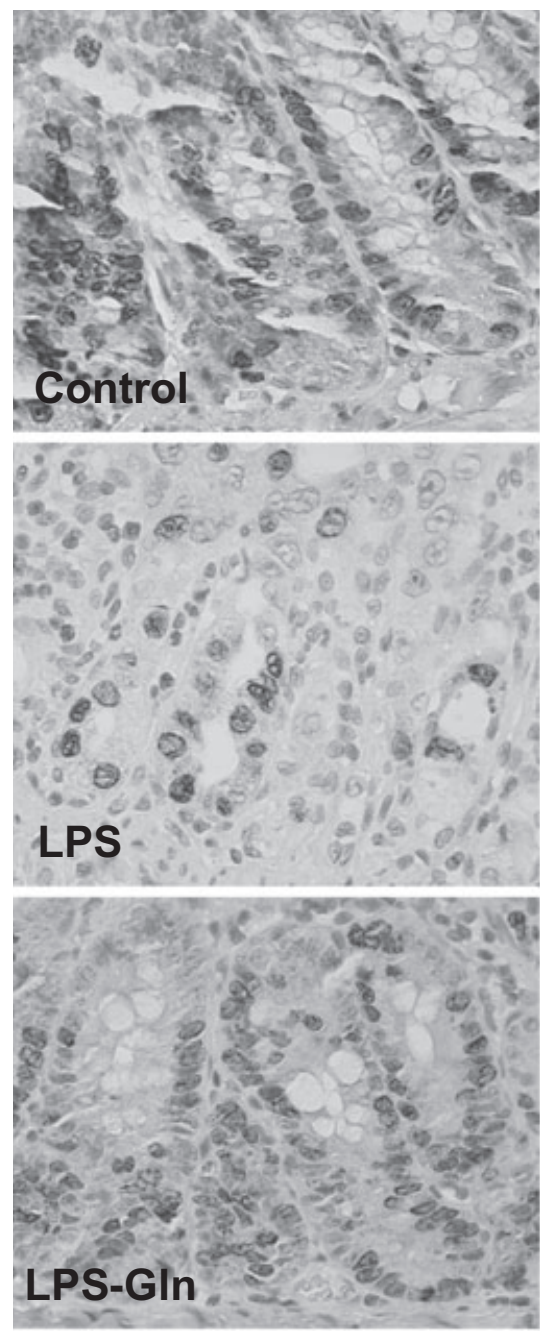

Apoptosis
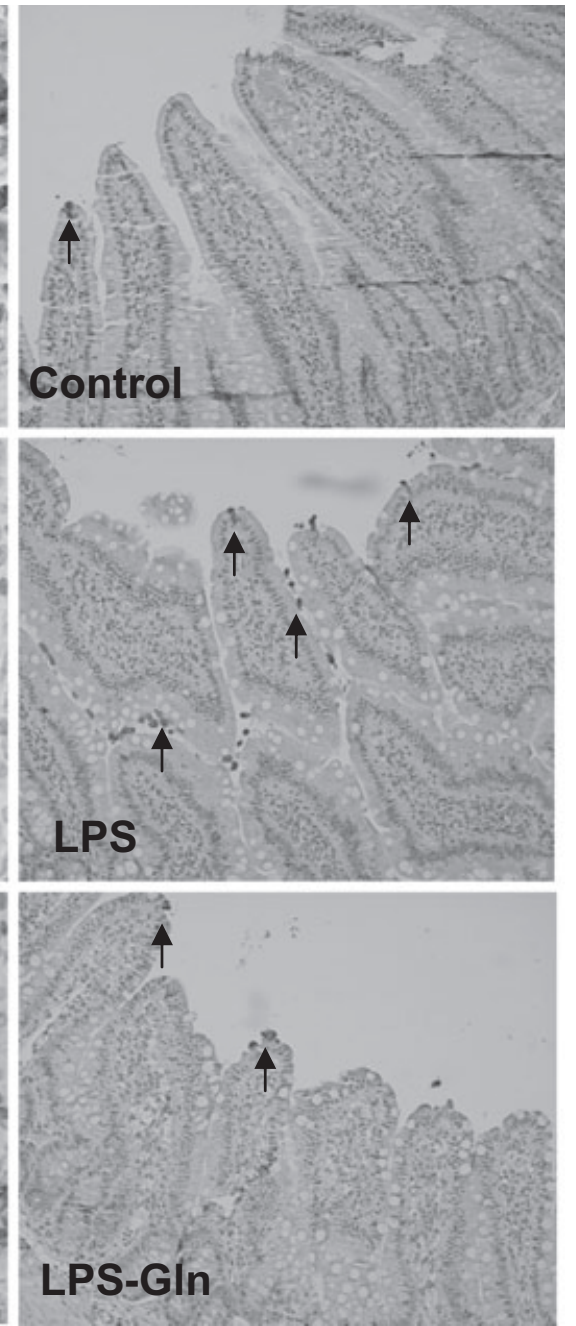


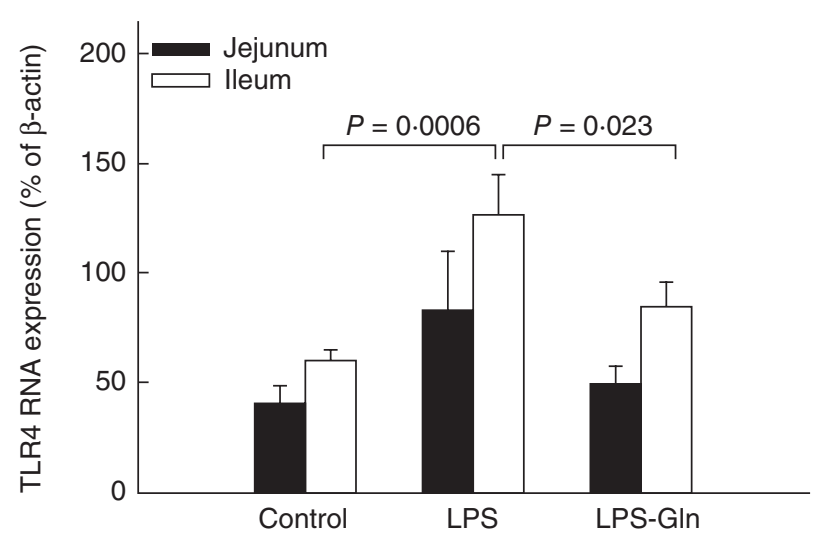

Fig. 2. Toll-like receptor-4 (TLR-4) mRNA expression levels in the intestinal mucosa. Following lipopolysaccharide (LPS) administration, the expression of TLR- 4 mRNA expression increased significantly compared to the control group in the ileal mucosa $(P=0.0006)$. Pretreatment with oral glutamine (LPS-Gln) decreased the elevation of TLR-4 mRNA expression significantly compared to the LPS group in the ileal mucosa $(P=0 \cdot 023)$. Similar results without statistical significance were found in the jejunal mucosa. Data shown are mean \pm standard error of the mean.

trend towards a decrease in the jejunum; however, this trend was not statistically significant. LPS rats (group B) showed a significant increase in TRAF6 mRNA expression versus controls $(P=0.03)$ in ileum and not in the jejunum (Fig. 4). Treatment with Gln (group C) resulted in a significant decrease in ileal TRAF6 mRNA expression (versus LPS rats, group B) $(P=0 \cdot 035)$.

Interestingly, the TLR-4, MyD88 and TRAF6 mRNA expression in the control groups was significantly higher in

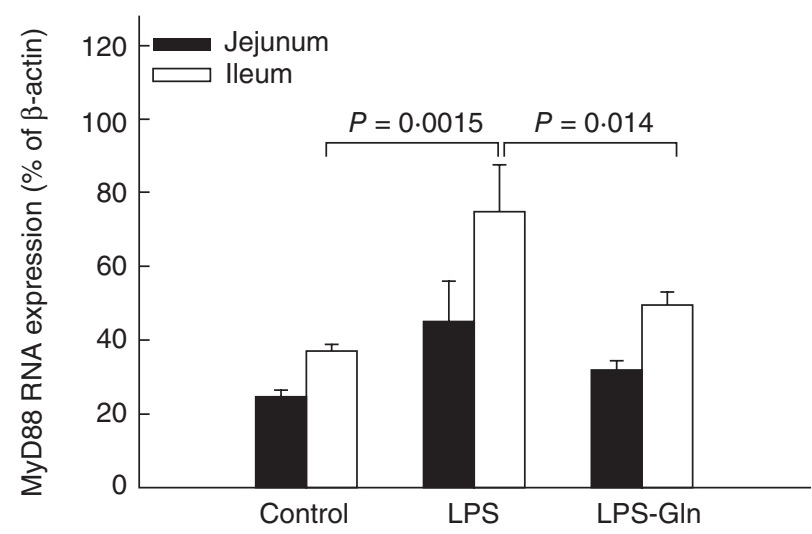

Fig. 3. Myeloid differentiation factor 88 (MyD88) mRNA expression levels in the intestinal mucosa. Following lipopolysaccharide (LPS) administration, the expression of MyD88 mRNA expression increased significantly compared to the control group in the ileal mucosa $(P=0 \cdot 0015)$. Pretreatment with oral glutamine (LPS-Gln) decreased the elevation of MyD88 mRNA expression significantly compared to the LPS group in the ileal mucosa $(P=0 \cdot 014)$. Similar observations without statistical significance were found in the jejunal mucosa. Data shown are mean \pm standard error of the mean. the ileum compared to the jejunum $(P=0.05, P=0.00019$ and $P=0 \cdot 016$, respectively).

\section{Expression of TLR-4 and MyD88 proteins}

Western blot analysis confirmed the changes in TLR-4 and MyD88 (Fig. 5). In sham segments, the expression of TLR-4 and MyD88 proteins was consistently higher in jejunal compared to ileal segments $(P<0 \cdot 05)$. A significant increase in expression of TLR- 4 and MyD88 was found in ileum of LPS rats compared to sham animals $(P<0 \cdot 05)$. Treatment with Gln resulted in a significant decrease in TLR-4 $(P<0.05)$ and MyD88 $(P<0.05)$ protein expression in ileum compared to LPS non-treated animals (group B).

\section{Discussion}

The intestinal epithelium serves as an important defence against luminal bacteria $[19,20]$. One of the mechanisms by which the intestinal epithelium controls the microflora and responds more actively to encroaching pathogens is referred to as the innate intestinal epithelial defence and is the TLR system. TLR-4 mRNA is present in mouse and human intestinal epithelial cells TLR-4 lines [21]. TLR-4 is also expressed on macrophages, dendritic cells, airway epithelia, endothelial cells and smooth muscle [22,23].

Involvement of TLR-4 in LPS responses was first suggested by the discovery that TLR-4 from spontaneous LPShyporesponsive mutant mice, $\mathrm{C} 3 \mathrm{H} / \mathrm{HeJ}$, has a point mutation that causes an inability to activate NFKB [24-26]. Subsequently, using generated TLR-4-deficient mice, TLR-4 was demonstrated unequivocally to be involved in LPS

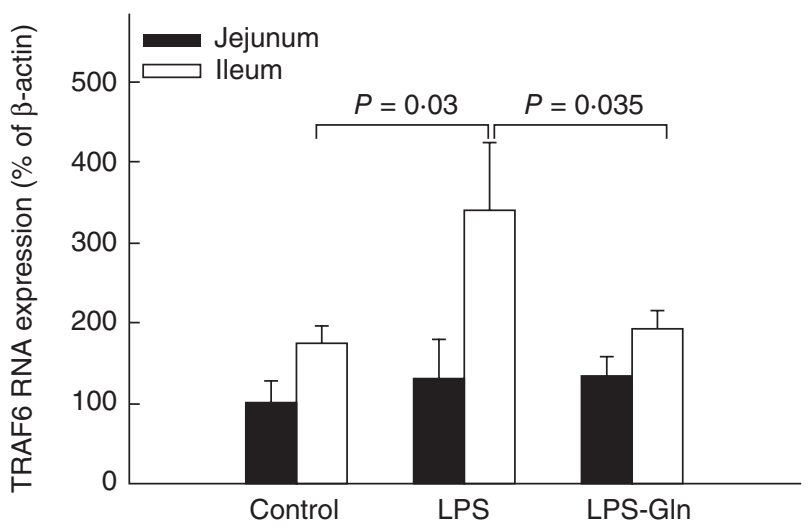

Fig. 4. Tumour necrosis factor (TNF)- $\alpha$ receptor-associated factor 6 (TRAF6) mRNA expression levels in the intestinal mucosa. Following lipopolysaccharide (LPS) administration, the expression of TRAF6 mRNA expression increased significantly compared to the control group in the ileal mucosa $(P=0 \cdot 03)$. Pretreatment with oral glutamine (LPS-Gln) decreased the elevation of TRAF6 mRNA expression significantly compared to the LPS group in the ileal mucosa $(P=0 \cdot 035)$. We did not find similar results in the jejunal mucosa. Data shown are mean \pm standard error of the mean. 

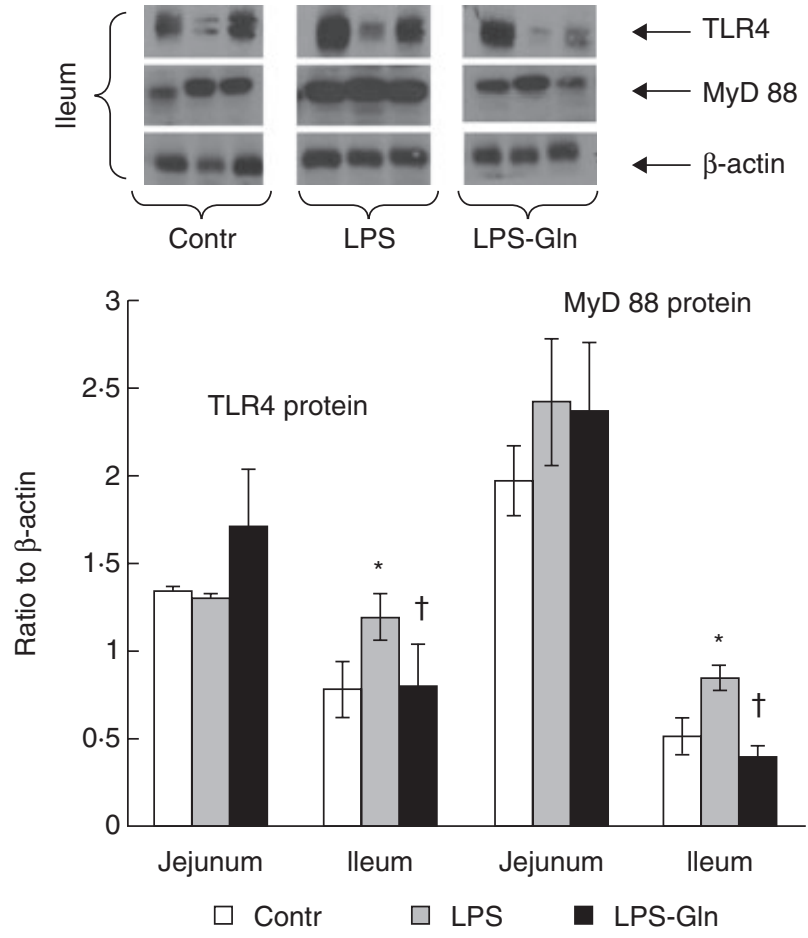

Fig. 5. Protein expression of Toll-like receptor-4 (TLR-4) and myeloid differentiation factor 88 (MyD88) in lipopolysaccharide (LPS) rats compared to control animals. $\beta$-actin was used to normalize loading. LPS rats demonstrate a significant increase in TLR- 4 and MyD88 expression in ileum compared to control animals. Protein expression of TLR-4 and MyD88 decreased significantly in ileum following glutamine administration. Data shown are mean \pm standard error of the mean. ${ }^{\star} P<0.05$ versus control; $\nmid$ LPS-Gln versus LPS.

responses [26]. While TLR-4 recognition of LPS is required for clearance of Gram-negative organisms, it is believed that the degree and the duration of this proinflammatory cytokine secretion can become harmful to the host [27-29]. The LPS receptor complex consists of two interacting receptors (CD14 and TLR-4) and an associated protein (MD-2). When engaged by LPS, as in Gram-negative infection, this complex transduces a signal detected by MyD88, which is passed onwards by a cascade of the receptor-associated kinase (IRAKs), receptor-associated factor 6 (TRAF6) and NFKBinducing kinase (NIK), resulting in activation of NFKB. In a recent experiment, we have shown that LPS endotoxaemia causes a significant mucosal hypoplasia in a rat which resulted in decreased enterocyte proliferation and increased apoptosis $[7,8]$. In this study we present a similar observation that demonstrates decreased mucosal weight, decreased mucosal DNA, decreased enterocyte proliferation and increased enterocyte apoptosis in rat intestine following LPS administration.

Analysis of gene and protein expression of selected members of the TLR-5 pathway revealed that their expression profiles vary greatly along the proximal-distal axis of the gut. We found that administration of LPS was associated with up-regulation of TLR-4, Myd88 and TRAF6 mRNA expression in the ileal mucosa; however, in jejunum these changes were not statistically significant. In accordance with mRNA expression, TLR-4 and Myd88 protein expression were found to be up-regulated in ileum of LPS rats compared to control animals. The up-regulation expression of TLR-4 and Myd88, which leads to induction of inflammatory cytokines such as TNF- $\alpha$, IL-1 and IL-6, can explain the developed widespread intestinal mucosal injury. Similar findings were reported in a study conducted by William et al. [30]. The authors found a correlation between induction of sepsis by caecal ligation and puncture, early expression of TLR-4 and onset of animal death.

The present study demonstrates that enteral Gln improves intestinal recovery following LPS administration. This is evident from increased bowel and mucosal weight, mucosal DNA and index of proliferation, although cell apoptosis did not change significantly with dietary Gln supplementation. This positive effect of Gln on intestinal mucosa was accompanied by a decrease in the expression of TLR-4, Myd88 and TRAF6 mRNA in the mucosal intestine. Specifically, the decrease of TLR-4, Myd88 and TRAF6 mRNA expression as well as TLR-4 and Myd88 protein expression in LPS-Gln versus LPS was statistically significant in the ileum. In fact, we found that the expression of TLR-4, MyD88 and TRAF6 in the control group (group A) was higher in the ileum compared to the jejunum. This observation might explain the different response of the TLR-4, Myd88 and TRAF6 mRNA expression in the ileal compare to the jeunal mucosa following LPS administration. Interestingly, Uehara et al. also found that Gln exerts a higher expression of haem oxygenase 1 in the ileum than in the jejunum [17].

Expression of TLR-4, MyD88 and TRAF6 mRNA decreased after Gln administration, which coincided with a decrease in TLR-4 and MyD88 protein expression. This finding is consistent with the Abreu et al. study [28]. They suggested that this down-regulation may be a mechanism by which intestinal epithelial cells protect against dysregulated immune signalling in response to Gram-negative commensal bacteria and their products. In a recent study, Cario and Podolsky demonstrated that in patients with inflammatory bowel disease the TLR-4 expression was higher than in healthy controls. They concluded that aberrant TLR-4 expression may play an important role in the loss of tolerance to the enteric bacteria [21].

In summary, LPS endotoxaemia causes intestinal mucosal injury and increases TLR-4 MyD88 and TRAF6 mRNA expression. Gln improves intestinal recovery and decreases TLR-4, MyD88 and TRAF6 mRNA expression. We conclude, therefore, that TLR-4, MyD88 and TRAF6 expression may be responsible for the harmful effect of LPS and positive effect of Gln on intestinal recovery. The positive effect of Gln on intestinal structure after LPS endotoxaemia may be considered as a mechanism via which immunonutrition helps in the recovery of critically ill and septic patients. 


\section{References}

1 Medzhitov R. Toll-like receptors and innate immunity. Nature reviews. Immunology 2001; 1:135-45.

2 Akira S, Takeda K. Toll-like receptor signalling. Nat Rev Immunol 2004; 4:499-511.

3 Poltorak A, He X, Smirnova I et al. Defective LPS signaling in $\mathrm{C} 3 \mathrm{H} / \mathrm{HeJ}$ and $\mathrm{C} 57 \mathrm{BL} / 10 \mathrm{ScCr}$ mice: mutations in Tlr4 gene. Science 1998; 282:2085-8.

4 Beutler B. Endotoxin, Toll-like receptor 4, and the afferent limb of innate immunity. Curr Opin Microbiol 2000; 3:23-8.

5 Medzhitov R, Preston-Hurlburt P, Janeway CA Jr. A human homologue of the Drosophila Toll protein signals activation of adaptive immunity. Nature 1997; 388:394-7.

6 Deitch EA. The role of intestinal barrier failure and bacterial translocation in the development of systemic infection and multiple organ failure. Arch Surg 1991; 125:403-9.

7 Sukhotnik I, Mogilner J, Krausz MM et al. Oral arginine reduces gut mucosal injury caused by lipopolysaccharide endotoxemia in a rat. J Surg Res 2004; 122:256-62.

8 Lacey JM, Wilmore DW. Is glutamine a conditional essential amino-acid? Nutr Rev 1990; 48:297-309.

9 Roth E, Funovics J, Mühlbacher F et al. Metabolic disorder in severe abdominal sepsis: glutamine deficiency in skeletal muscle. Clin Nutr 1982; 1:25-41.

10 Gamrin L, Essen P, Forsberg AM et al. A descriptive study of skeletal muscle metabolism in critically ill patients: free amino acids, energy-rich phosphates, protein, nucleic acids, fat, water, and electrolytes. Crit Care Med 1996; 24:575-83.

11 Houdijk AP, Rijnsburger ER, Jansen J et al. Randomised trial of glutamine-enriched enteral nutrition on infectious morbidity in patients with multiple trauma. Lancet 1998; 352:772-6.

12 Novak F, Heyland DK, Avenell A et al. Glutamine supplementation in serious illness: a systematic review of the evidence. Crit Care Med 2002; 30:2022-9.

13 Tremel H, Kienle B, Weilemann LS et al. Glutamine dipeptidesupplemented parenteral nutrition maintains intestinal function in the critically ill. Gastroenterology 1994; 107:1595-601.

14 Zapata-Sirvent RL, Hansbrough JF, Ohara MM et al. Bacterial translocation in burned mice after administration of various diets including fiber- and glutamine-enriched enteral formulas. Crit Care Med 1994; 22:690-6.

15 Preiser JC, Wernerman J. Glutamine, a life-saving nutrient, but why? Crit Care Med 2003; 31:2555-6.

16 Uehara K, Takahashi T, Fujii $\mathrm{H}$ et al. The lower intestinal tractspecific induction of heme oxygenase- 1 by glutamine protects against endotoxemic intestinal injury. Crit Care Med 2005; 33:38190.

17 Sukhotnik I, Agam M, Shamir R et al. Oral glutamine prevents gut mucosal injury and improves mucosal recovery following lipopolysaccharide endotoxemia in a rat. J Surg Res 2007; 143: 379-84.

18 Chomczynski P. A reagent for the single-step simultaneous isolation of RNA, DNA and proteins from cell and tissue samples. Biotechniques 1993; 15:532-7.

19 Hecht G. Innate mechanisms of epithelial host defense: spotlight on intestine. Am J Physiol 1999; 277:C351.

20 Kim J, Eckmann ML, Savidge TC, Lowe DC, Witthoft T, Kagnoff MF. Apoptosis of human intestinal epithelial cells after bacterial invasion. J Clin Invest 1998; 102:1815-23.

21 Cario E, Rosenberg IM, Brandwein SL, Beck PL, Reinecker HC, Podolsky DK. Lipopolysaccharide activates distinct signaling pathways in intestinal epithelial cell lines expressing Toll-like receptors. J Immunol 2000; 164:966-72.

22 Zhang FX, Kirschning CJ, Mancinelli R et al. Bacterial lipopolysaccharide activates nuclear factor- $\kappa \mathrm{B}$ through interleukin-1 signaling mediators in cultured human dermal endothelial cells and mononuclear phagocytes. J Biol Chem 1999; 274:7611-4.

23 Muzio M, Bosisio D, Polentarutti N et al. Differential expression and regulation of Toll-like receptors (TLR) in human leukocytes: selective expression of TLR3 in dendritic cells. J Immunol 2000; 164:5998-6004.

24 Poltorak A, He X, Smirnova I et al. Defective LPS signaling in $\mathrm{C} 3 \mathrm{H} / \mathrm{HeJ}$ and $\mathrm{C} 57 \mathrm{BL} / 10 \mathrm{ScCr}$ mice: mutations in Tlr4 gene. Science 1998; 282:2085-8.

25 Qureshi S, Lariviere TL, Leveque G et al. Endotoxin-tolerant mice have mutations in Toll-like receptor 4 (Tlr4). J Exp Med 1999; 189:615-25.

26 Hoshino K, Takeuchi O, Kawai T et al. Cutting edge: Toll-like receptor 4 (TLR4)-deficient mice are hyporesponsive to lipopolysaccharide: evidence for TLR4 as the lps gene product. J Immunol 1999; 162:3749-52.

27 Medvedev AE, Kopydlowski KM, Vogel SN. Inhibition of lipopolysaccharide-induced signal transduction in endotoxintolerized mouse macrophages: dysregulation of cytokine, chemokine, and Toll-like receptor 2 and 4 gene expression. J Immunol 2000; 164:5564-74.

28 Abreu MT, Vora P, Faure E, Thomas LS, Arnold ET, Aarditi M. Decreased expression of Toll-like receptor-4 and MD-2 correlates with intestinal epithelial cell protection against dysregulated proinflammatory gene expression in response to bacterial lipopolysaccharide. J Immunol 2001; 167:1609-16.

29 Bosisio D, Polentarutti N, Sironi M et al. Stimulation of Tolllike receptor 4 expression in human mononuclear phagocytes by interferon-gamma: a molecular basis for priming and synergism with bacterial lipopolysaccharide. Blood 2002; 99:342731.

30 William DL, Ha T, Li C et al. Modulation of tissue Toll-like receptor 2 and 4 during the early phases of polymicrobial sepsis correlates with mortality. Crit Care Med 2003; 31:1808-18. 